\title{
Taux d'administration du vaccin contenant le virus de la rougeole dans le Sud de l'Alberta
}

\author{
Matkin $\mathrm{A}^{1}, \mathrm{~K}$ Simmonds ${ }^{2}$ et V Suttorp ${ }^{3^{*}}$ \\ Faculty of Health Sciences, University of Lethbridge, Lethbridge (Alberta) \\ 2 Surveillance and Assessment Branch, ministère de la Santé de l'Alberta, Edmonton (Alberta) \\ 3 Population and Public Health, Alberta Health Services, Lethbridge (Alberta) \\ * Auteur-ressource : vivien.suttorp@albertahealthservices.ca
}

\section{Résumé}

Contexte : Le Sud de l'Alberta est habité par de nombreuses collectivités uniques et homogènes qui envoient généralement leurs enfants dans des écoles privées. Pour diverses raisons, nombre de ces collectivités ne font pas la promotion de la vaccination comme mesure de santé publique de prévention. Les membres de ces collectivités se tiennent plutôt à l'écart des autres populations et, par conséquent, ne bénéficient pas de l'immunité collective générale. Cette situation a souvent causé des éclosions de maladies évitables par la vaccination dans les écoles privées associées à ces communautés religieuses homogènes.

Objectif : Rendre compte des faibles taux de vaccination ROR (rougeole, oreillons et rubéole) et de vaccination ROR-V (ROR et varicelle) dans les collectivités et les écoles du Sud de l'Alberta, et comparer l'épidémiologie des taux de vaccination de certaines collectivités vulnérables à ceux de cohortes des mêmes âges dans les collectivités de la zone sud.

Méthodes : L'analyse porte sur des données relatives à la vaccination des personnes consignées au registre provincial d'immunisation, au registre Immunization and Adverse Reactions to Immunization et au registre de l'Alberta Health Services par l'intermédiaire du module Meditech, du 1 ${ }^{\mathrm{er}}$ janvier 2013 au 30 juin 2013.

Résultats : On a décelé une hétérogénéité de l'état vaccinal parmi les collectivités et les écoles. La proportion d'enfants de 2 ans dont la vaccination était à jour variait de 46,6 \% à Fort Macleod à 71,9\% à Oyen, avec une moyenne de 57,3 pour 100 enfants qui étaient à jour. Dans le Sud de l'Alberta, le pourcentage moyen d'enfants vaccinés à l'âge de 7 ans était de $77,6 \%$ et variait de $57,8 \%$ à Picture Butte à $94,6 \%$ à Oyen. Le taux de vaccination dans les écoles variait de 17 à $100 \%$, avec une moyenne de 89,3\% d'enfants ayant reçu toutes les doses et une médiane de $91 \%$ d'enfants vaccinés.

Conclusion : Parmi les collectivités et les écoles du Sud de l'Alberta, on observe une hétérogénéité de l'immunisation des enfants par le vaccin contenant le virus de la rougeole. Cette étude met en lumière le fait que le taux de vaccination observé dans une école peut ne pas être en corrélation avec celui observé dans la collectivité géographique. L'analyse des données concernant le taux de vaccination des enfants, tant à l'échelle des collectivités que des écoles, est importante en vue de comprendre les risques de propagation de maladies évitables par la vaccination dans une région donnée, comme celle de la zone sud de l'Alberta. Les résultats de cette étude peuvent servir à éclairer des interventions précises nécessaires à l'amélioration des taux de couverture vaccinale dans ces communautés culturelles homogènes uniques et dans leurs écoles, ainsi qu'à la réduction du risque de transmission de la rougeole dans le Sud de l'Alberta.

\section{Introduction}

La rougeole est une maladie hautement transmissible causée par le transfert direct du virus de la rougeole par des gouttelettes respiratoires ou par des aérosols en petites particules à des hôtes humains. Les particules restent en suspension dans l'air pendant une période allant jusqu'à deux heures et la meilleure façon de prévenir la transmission du virus est l'immunisation active par le vaccin contre la rougeole (1). Afin d'obtenir une immunité collective efficace contre la rougeole, le ministère de la Santé de l'Alberta a établi les taux de 
vaccination cibles de $98 \%$ chez les enfants âgés de 2 ans pour la première dose du vaccin contenant le virus de la rougeole et de $99 \%$ chez les enfants ayant atteint l'âge de 7 ans pour la deuxième dose de ce vaccin (2). La prévention de la transmission dépend toujours de nombreux facteurs, notamment d'un vaccin hautement immunogène, du mélange aléatoire d'une population hétérogène et d'une couverture vaccinale uniforme d'un groupe à l'autre (3). Le vaccin contre la rougeole est hautement efficace, car une seule dose procure une immunité variant de 85 à $95 \%$ et une dose de rappel en accroît l'efficacité, qui atteint alors près de $100 \%$ (4). Dans une population donnée, certaines personnes choisissent de ne pas se faire vacciner. Le concept de mélange aléatoire fait allusion à l'importance que ces personnes non vaccinées soient incorporées dans la portion de la population qui est vaccinée, où elles peuvent être protégées contre la maladie grâce à l'immunité collective. L'immunité collective protège les collectivités contre une éclosion de rougeole en empêchant la propagation rapide du virus. Bien que le nombre d'enfants infectés par le virus de la rougeole et qui meurent de cette maladie chaque année ait considérablement chuté depuis l'avènement de l'administration systématique du vaccin contre la rougeole, cette maladie demeure un problème de santé publique et elle réapparaît dans des pays où l'on croyait qu'elle était éliminée ou presque éliminée (1,5-7). De fermes convictions religieuses ou culturelles en défaveur de la vaccination, l'augmentation des voyages dans des pays et sur des continents étrangers, des sous-populations homogènes dont la couverture vaccinale est insuffisante et le manque de renseignements ou la désinformation à propos de la sécurité des vaccins contribuent à la réapparition de la rougeole $(3,7)$.

La partie la plus au sud de l'Alberta - la région située au sud de Calgary - est l'une des cinq zones géographiques de l'Alberta où les soins de santé sont dispensés par l'intermédiaire de l'Alberta Health Services. Dans la zone sud, on compte 17 bureaux de santé publique responsables de la vaccination des enfants. Dans la zone sud, se trouvent diverses collectivités socialement isolées, dans lesquelles le taux de vaccination est faible pour différentes raisons (8). La plus importante de ces collectivités est formée de la Netherlands Reformed Congregation, de collectivités mennonites s'exprimant en bas-allemand et de quelques colonies huttériennes. Les membres de chacune de ces communautés partagent des convictions religieuses ou culturelles uniques; ils sont étroitement liés à leur communauté et, de ce fait, ne se mélangent aléatoirement que de façon minimale au reste de la population de leur zone géographique. La majorité des enfants de ces collectivités sont transportés en autobus scolaires vers des écoles privées ou reçoivent leur enseignement scolaire à la maison et participent à des activités parascolaires, notamment des activités sportives et religieuses, à l'intérieur de leurs réseaux sociaux très unis. Les membres de ces collectivités entretiennent des liens étroits avec des pays ou des régions comme les Pays-Bas, le Mexique et l'Amérique du Sud, ainsi qu'avec des peuplements similaires situés partout en Amérique du Nord. Les voyages vers les communautés liées dans lesquelles les taux de vaccination sont faibles posent un risque d'importation de maladies évitables par la vaccination dans le Sud de l'Alberta et un risque potentiel élevé de propagation. Bien que quelques membres et familles de ces communautés religieuses conservatrices commencent à accepter la vaccination, beaucoup ne font toujours pas vacciner leurs enfants. Les normes et les perspectives culturelles compliquent la prise de décisions éclairées des membres de ces collectivités à propos de la vaccination (8-10).

Les éclosions de maladies évitables par la vaccination sont, depuis longtemps, fréquentes dans le Sud de l'Alberta. Par exemple, au cours des 20 dernières années, des éclosions de coqueluche se sont produites tous les trois à cinq ans. Les dernières grandes éclosions de coqueluche observées dans cette région se sont produites en 2009 et en 2012, dans différentes communautés religieuses. L'analyse de l'éclosion de 2009 a révélé que la source de cette éclosion se trouvait dans une des écoles privées dont les taux de vaccination étaient faibles et que la transmission de la maladie s'est poursuivie sur une période de 11 mois à l'intérieur de cette école. Deux mois après le début de l'éclosion, la coqueluche s'est propagée dans les écoles publiques des collectivités géographiques dans lesquelles les enfants de l'école de référence habitaient. Comme les taux de vaccination étaient plus près du seuil d'immunité collective cible dans les écoles publiques de la collectivité, la transmission au sein de ces écoles a été minimale.

L'objectif du présent article consiste à rendre compte des faibles taux de vaccination ROR et de vaccination ROR-V dans les collectivités et les écoles du Sud de l'Alberta et à comparer l'épidémiologie des taux de vaccination dans certaines collectivités vulnérables à ceux de cohortes des mêmes âges dans les collectivités de la zone sud. Des analyses des taux de vaccination dans les zones géographiques recevant les 
services de bureaux locaux de santé publique permettront de déterminer quelles zones présentent les taux de vaccination les plus élevés et les plus faibles, puis les conclusions guideront les mesures futures visant à établir les meilleures méthodes d'intervention et d'éducation.

\section{Méthodes}

Au Canada, l'immunisation contre la rougeole, les oreillons, la rubéole et, plus récemment, la varicelle est dispensée par un seul vaccin (ROR ou ROR-V), administré en deux doses (11). En Alberta, l'ensemble de la vaccination des enfants est effectué par l'organisme de santé publique Alberta Health Services, dans des bureaux de santé publique et les écoles, et le ministère de la Santé de l'Alberta est responsable des politiques et des directives en matière d'immunisation. Selon le calendrier de vaccination systématique du ministère de la Santé de l'Alberta, les enfants de l'Alberta reçoivent leur première dose du vaccin à l'âge de 1 an et leur seconde, à l'âge de 4, 5 ou 6 ans (11). Les données individuelles sur la vaccination dans la zone sud de l'Alberta sont consignées au point de service, par l'intermédiaire du module Meditech sur la vaccination. Les données saisies dans le module Meditech sont transmises au registre provincial du ministère de la Santé de l'Alberta, I'Imm/ARI (pour "Immunization and Adverse Reactions to Immunization »). Aux fins de la présente étude, les données sur la vaccination au sein des collectivités proviennent d'une interrogation de la banque de données Imm/ARI en fonction des codes postaux et les données portant sur la vaccination dans les écoles proviennent de Meditech. Seuls les enfants fréquentant les écoles publiques et privées ont été inclus; les enfants recevant leur enseignement scolaire à la maison n'ont pas été compris dans l'analyse. Les écoles ont été regroupées en fonction du code postal que les bureaux de santé publique utilisent afin d'analyser les données sur la vaccination dans les collectivités. On a qualifié les enfants âgés de 2 ans n'ayant reçu aucune dose du vaccin comme étant non vaccinés; on a considéré que la vaccination des enfants de cet âge ayant reçu une dose du vaccin était à jour. On a considéré les enfants âgés de 7 ans n'ayant reçu aucune dose du vaccin (non immunisés) comme faisant possiblement partie d'une famille dont les parents refusent la vaccination; les enfants de cet âge ayant reçu une seule dose ont été caractérisés comme étant partiellement immunisés et ceux ayant reçu deux doses (vaccination à jour) comme étant entièrement immunisés.

Les données sur la vaccination des enfants qui avaient été vaccinés dans d'autres régions de l'Alberta ou à l'extérieur de cette province et dont les dossiers n'avaient pas encore été mis à jour n'ont pas été saisies avec exactitude dans la présente analyse. II s'agissait entre autres d'enfants des Premières nations qui avaient reçu leurs vaccins dans le cadre de programmes administrés par le gouvernement fédéral. Compte tenu de cette situation, les données sur la vaccination des villes de Standoff et de Brocket, situées dans deux collectivités de Premières nations, n'ont pas été incluses dans l'analyse. En outre, les taux de vaccination de Cardston doivent être interprétés avec prudence, car les codes postaux de cette municipalité comprennent des zones où les enfants reçoivent leurs vaccins dans le cadre d'un programme fédéral.

\section{Analyse des données}

Les taux de vaccination pour 100 habitants d'une collectivité ont été calculés à partir des dénominateurs et des données démographiques accessibles dans l'Imm/ARI. On a utilisé les données des bureaux de santé publique sur les populations d'enfants âgés de 2 et de 7 ans le 30 juin 2013. Les âges de 2 et de 7 ans ont été choisis en fonction du calendrier de vaccination systématique (9); ces âges offrent une latitude d'un an pour que les enfants puissent recevoir leurs doses à temps. Nous avons comparé le nombre de doses du vaccin que les enfants avaient reçues à l'âge de 2 ans (aucune dose ou au moins une dose) et à l'âge de 7 ans (aucune dose, une dose ou au moins deux doses) dans la zone d'attraction de chaque bureau de santé publique. Le nombre d'enfants de chaque cohorte (âgés de 2 ans et de 7 ans) ayant reçu aucune, une ou deux doses a été inclus dans le numérateur et le nombre total d'enfants de chaque groupe d'âge était le dénominateur. Le quotient a été multiplié par 100 afin d'obtenir l'incidence pour 100 enfants. Les taux de vaccination en fonction de l'école ont été calculés de la même manière, à partir des données du module Meditech portant sur les enfants fréquentant des écoles publiques et privées qui étaient âgés de 7 ans le 30 juin 2013. 


\section{Résultats}

Les résultats indiquent que, le 30 juin 2013, 42,8\% des enfants âgés de 2 ans de la zone sud de l'Alberta Health Services étaient non vaccinés et que la vaccination de $57,3 \%$ de cette population d'enfants était à jour. Au même moment, $14,3 \%$ des enfants âgés de 7 ans n'étaient pas vaccinés, $8,1 \%$ n'avaient reçu qu'une dose du vaccin et $77,6 \%$ en avaient reçu les deux doses. Les taux de vaccination en fonction de l'âge sont illustrés dans les Figures 1 et 2.

Figure 1. Taux de vaccination des enfants âgés de 2 et de 7 ans dans la zone sud de l'Alberta Health Services

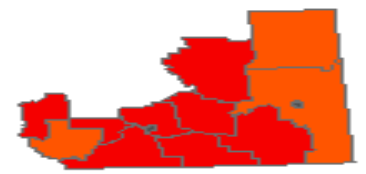

(a) Vaccination contre la rougeole, une dose, 30 juin 2013 , à 2 ams
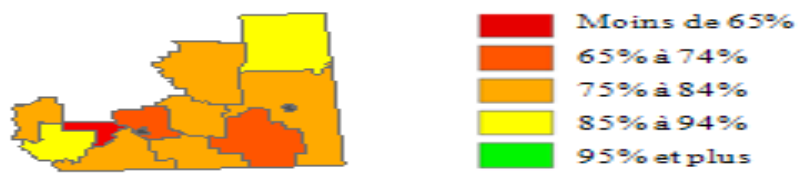

(b) Vaccination contre la rougeole, deux doses, 30 juin 2013 , à 7 ans

Figure 2. Taux d'incidence de la vaccination à 7 ans pour 100 enfants parmi la population admissible dans la zone d'attraction du bureau de santé publique de la zone sud de l'Alberta Health Services

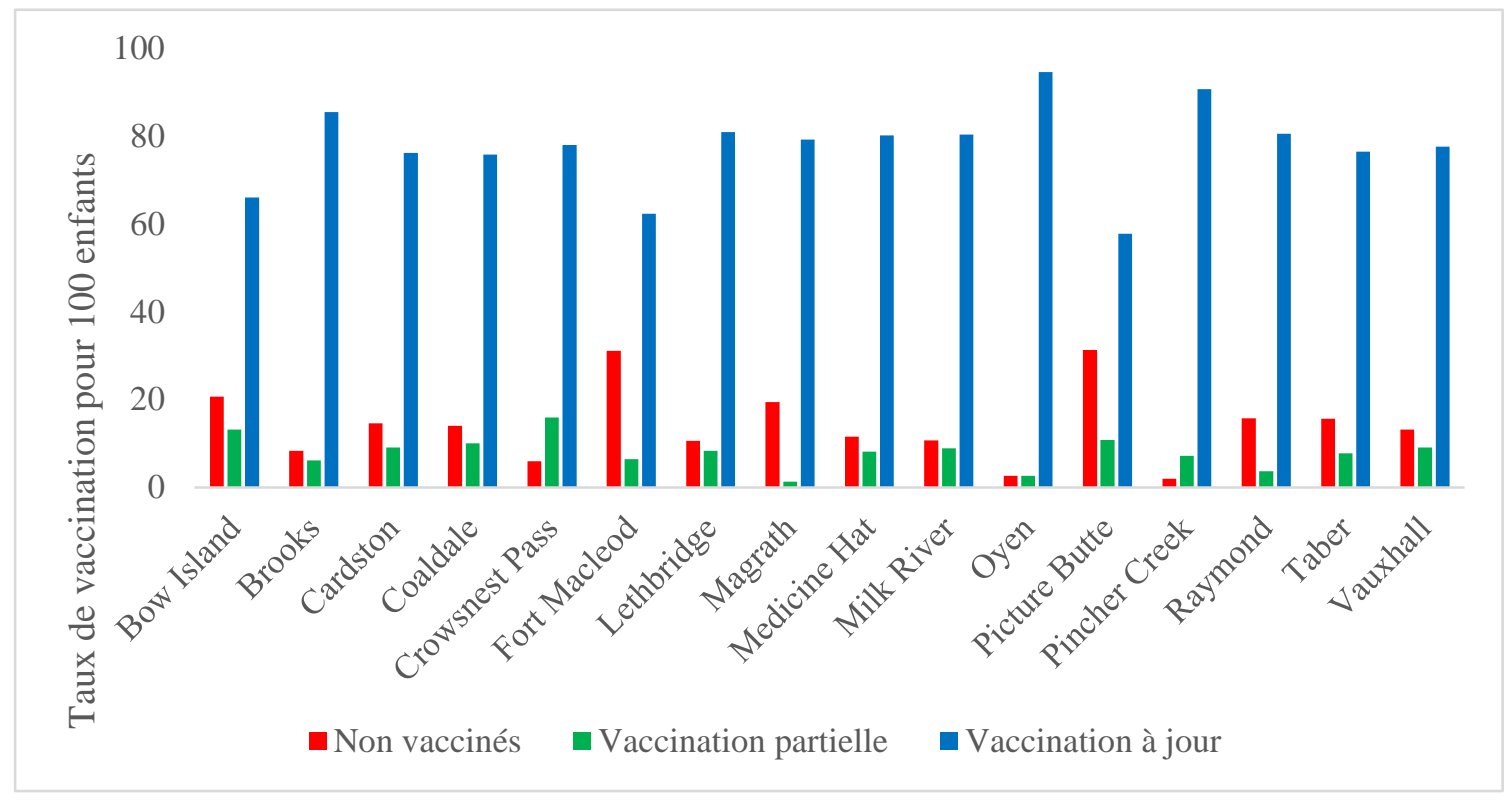

Lors de l'examen des taux de vaccination en fonction de la zone géographique des bureaux de santé publique, nous avons découvert que plus de la moitié des enfants âgés de 2 ans de Fort Macleod $(53,4 \%)$, de 
Picture Butte $(64,9 \%)$ et de Vauxhall $(59,5 \%)$ n'était pas vaccinée. Le taux d'incidence le plus élevé d'enfants dont la vaccination était à jour à l'âge de 2 ans était à Oyen (71,9\%) et la majorité du taux de vaccination complète parmi les bureaux de santé publique variait de 56,0\%, à Raymond, à 69,8\%, à Medicine Hat. Le taux d'incidence d'enfants non vaccinés à l'âge de 7 ans était élevé à Bow Island (20,8 \%), à Fort Macleod $(31,2 \%)$ et à Picture Butte (31,3\%). Les zones de ces bureaux de santé publique présentaient également de faibles taux d'enfants âgés de 7 ans ayant reçu toutes les doses du vaccin, soit 66,0,62,3 et 57,8\%, respectivement. Les taux d'incidence d'enfants ayant reçu toutes les doses du vaccin étaient supérieurs à $90 \%$ dans seulement deux bureaux de santé publique, soit Pincher Creek (90,7\%) et Oyen (94,6\%). La vaccination était à jour chez plus de $80 \%$ des enfants âgés de 7 ans de Brooks, Lethbridge, Medicine Hat, Milk River et Raymond.

Les écoles comptant des populations égales ou inférieures à 5 enfants âgés de 7 ans ont été omises, ainsi que l'école Ralston, du bureau de santé publique de Medicine Hat, pour un total de 80 écoles accessibles aux fins d'analyse. Oyen a été retiré de l'analyse, car toutes les écoles sous la responsabilité de ce bureau de santé publique comptaient moins de cinq enfants âgés de 7 ans. L'incidence moyenne de la vaccination partielle était de 94,4 enfants pour 100 admissibles (ÉT $=11,1$ ), avec une incidence médiane de vaccination de $100 \%$ et un intervalle de 17 à $100 \%$. Les résultats portant sur l'incidence des enfants ayant reçu toutes les doses du vaccin étaient similaires, avec une moyenne de $90 \%$ du taux de vaccination (ÉT = 12,1). À $91 \%$, le taux médian des enfants ayant reçu toutes les doses du vaccin dans l'ensemble des écoles était inférieur à celui des enfants partiellement vaccinés, mais l'intervalle était le même (de 17 à $100 \%$ ). Selon les résultats de l'analyse en fonction du bureau de santé publique, Fort Macleod $(n=1)$, Lethbridge $(n=23$; intervalle de 17 à $100 \%)$, Vauxhall $(n=2$; intervalle de 83 à $88 \%)$ et Picture Butte $(n=4$; intervalle de 78 à $100 \%)$ présentaient les plus faibles taux moyens de vaccination complète, soit $83,0 \% ; 83,1 \% ; 85,5 \%$ et $88,3 \%$, respectivement). Les résultats sont présentés à la Figure 3.

Figure 3. Taux d'incidence de la vaccination à 7 ans pour 100 enfants parmi la population admissible dans les écoles publiques et privées des zones d'attraction des bureaux de santé publique de la zone sud de l'Alberta Health Services

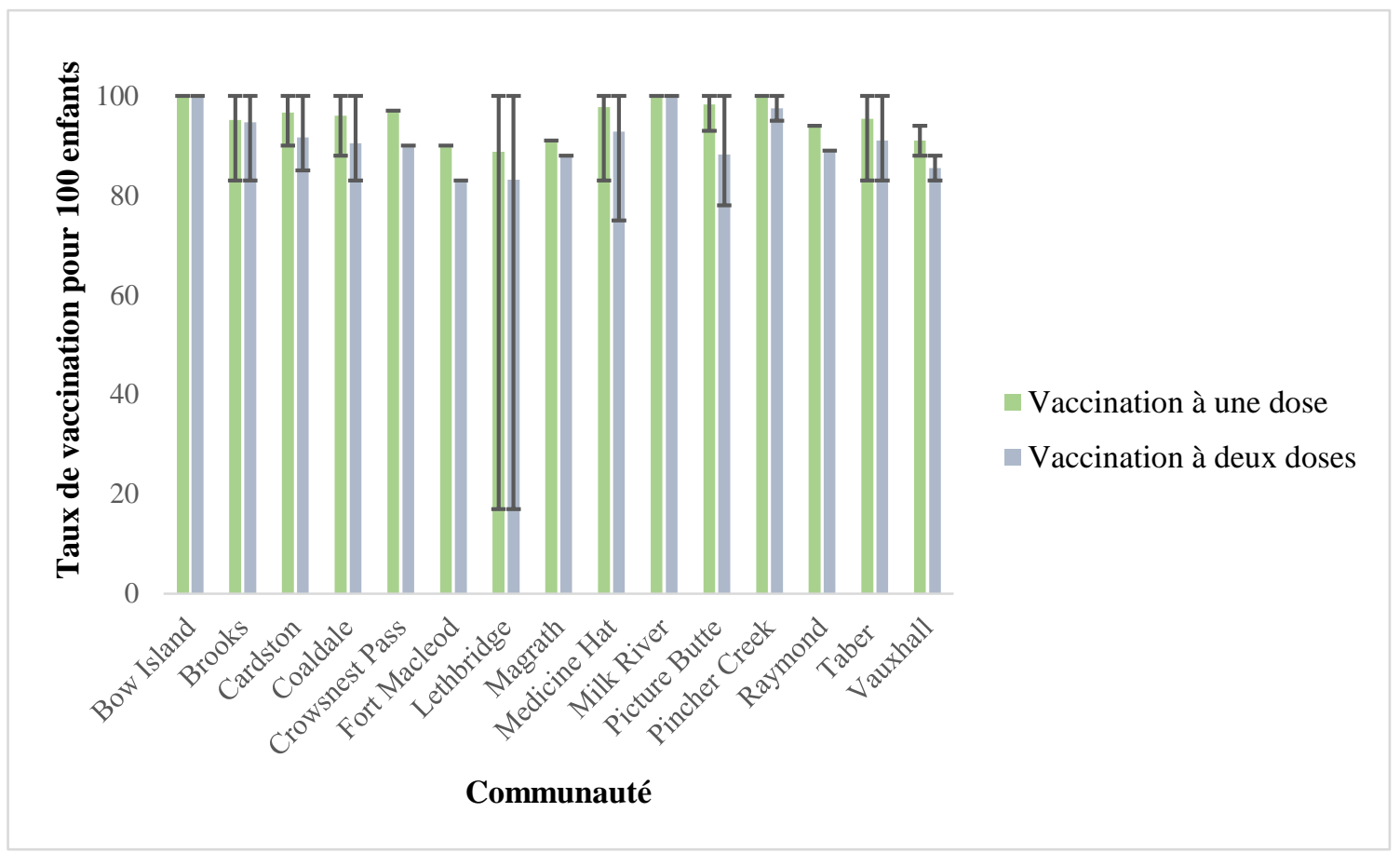

Les lignes représentent le taux moyen de vaccination dans les écoles en fonction des bureaux de santé publique; les barres d'erreur représentent l'intervalle de l'état vaccinal dans les écoles 


\section{Discussion}

Les résultats de cette étude révèlent que, dans la majorité des collectivités de la zone sud, la couverture par le vaccin contenant le virus de la rougeole est bien en deçà du taux de 90 à $95 \%$ accepté et requis pour obtenir une immunité collective et bien inférieure aux cibles pour la population de l'Alberta $(3,5)$. Nous avons découvert que seulement deux collectivités du Sud de l'Alberta ont un taux de vaccination qui approche la cible de $99 \%$ du ministère de la Santé de l'Alberta pour les enfants âgés de 7 ans. Un examen plus approfondi des taux de vaccination contre la rougeole dans les écoles révèle une importante hétérogénéité parmi les écoles, relativement à cette même cohorte des enfants de 7 ans. Les taux de vaccination contre la rougeole dans les écoles ne correspondent pas aux taux de vaccination observés dans les collectivités respectives. Deux raisons expliquent principalement cette situation. Premièrement, les écoles présentant un faible taux de vaccination contre la rougeole sont principalement des écoles privées, fréquentées par des enfants provenant, par autobus scolaires, de communautés religieuses ou culturelles particulières dispersées dans de vastes zones géographiques. Par exemple, le taux de vaccination chez les enfants de 7 ans fréquentant les quatre écoles de la région de Picture Butte a une portée de $78,0 \%$ et une médiane de $87,5 \%$. Cette collectivité est également l'un des lieux de résidence des enfants qui fréquentent une école privée dans la zone d'attraction du bureau de santé publique de Lethbridge, dont le taux de vaccination est de $17 \%$ dans cette cohorte d'âges.

Deuxièmement, l'absence d'uniformité entre les données provenant des écoles et celles provenant des collectivités s'explique aussi par le fait que les données des collectivités incluent aussi des enfants recevant leur enseignement scolaire à la maison et qui peuvent être ou ne pas être vaccinés. Par exemple, dans un grand nombre de foyers-écoles tenus par des mennonites s'exprimant en bas-allemand, la majorité des enfants ne sont pas immunisés et les familles sont plutôt de passage. Ces écoles ne sont pas incluses dans l'analyse des données, car les organismes de santé publique ne reçoivent pas leurs listes d'enfants inscrits et n'y effectuent pas la vaccination.

La connaissance des taux de vaccination en fonction des collectivités et des écoles fournit des renseignements sur le risque de propagation de maladies évitables par la vaccination, comme la rougeole. Une école où le taux de vaccination est faible est un endroit propice à la survenue d'une éclosion (12). Si des enfants non immunisés sont exposés ou présentent des symptômes et passent de leur école à leur collectivité, le virus de la rougeole peut alors se propager aux personnes vulnérables dans cette collectivité. Comme la région du Sud ne compte pas de zone géographique dont le taux d'immunisation est assez élevé pour procurer une immunité à toute la population (Figure 3), il est davantage possible qu'un virus hautement contagieux, comme celui de la rougeole, se propage dans toute la zone sud et dans d'autres régions de la province $(3,13)$.

La présente étude n'enquête par sur les raisons des faibles taux de vaccination dans les collectivités ni dans les écoles, telles que les convictions religieuses, les lacunes en matière d'éducation ou l'accès à la vaccination. Cependant, ces renseignements pourraient servir conjointement avec les résultats d'études antérieures ayant exploré le point de vue des personnes refusant de recevoir des vaccins dans le Sud de l'Alberta afin de guider l'élaboration de méthodes d'intervention ciblées (8). En outre, on recommande d'effectuer de plus amples recherches afin de recenser des méthodes acceptables d'intervention à l'échelle des écoles visant les écoles privées dont les taux de vaccination sont faibles. Le suivi des variations des taux de vaccination dans le temps dans chaque collectivité et dans chaque école de la zone sud est essentiel à la mesure des effets des interventions.

\section{Conclusion}

Parmi les collectivités et les écoles du Sud de l'Alberta, on observe une hétérogénéité de l'immunisation des enfants par le vaccin contenant le virus de la rougeole. Cette étude met en lumière le fait que le taux de vaccination observé dans une école peut ne pas être en corrélation avec celui de sa collectivité géographique. L'analyse des données concernant le taux de vaccination des enfants, tant à l'échelle des collectivités que des écoles, est importante en vue de comprendre les risques de propagation de maladies évitables par la vaccination dans une région donnée, comme celle de la zone sud de l'Alberta. Les résultats de cette étude peuvent servir à éclairer des interventions précises nécessaires à l'amélioration des taux de couverture 
vaccinale dans ces communautés culturelles homogènes uniques et dans leurs écoles, ainsi qu'à la réduction du risque de transmission de la rougeole dans le Sud de l'Alberta.

\section{Références}

(1) Moss WJ, Griffin DE. Measles. The Lancet 2012; 379:153-164.

(2) Alberta Immunization Strategy 2007-2017.http://www.health.alberta.ca/documents/ImmunizationStrategy-07.pdf

(3) Rashid H, Khandaker G, Boy R. Vaccination and herd immunity: what more do we know? Curr Opin Infect Dis 2012; 25:243-249.

(4) Comité consultatif national de l'immunisation. Vaccins actifs (partie 4). In: Guide canadien d'immunisation. Ottawa : Agence de la santé publique du Canada, 2012.http://www.phac-aspc.gc.ca/publicat/ciggci/p04-meas-roug-fra.php

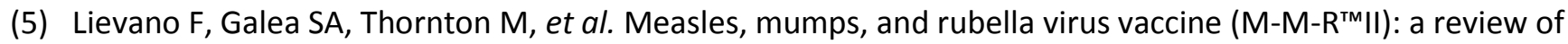
32 years of clinical and postmarketing experience. Vaccine 2012; 30(48):6918-6926.

(6) Leuridan E, Sabbe M, Van Damme P. Measles outbreak in Europe: susceptibility of infants too young to be immunized. Vaccine 2012; 30:5905-5913.

(7) Maltezou HC, Wicker S. Measles in health-care settings. Am J Infect Control 2013; 41:661-663.

(8) Kulig JC, Meyer CJ, Hill SA, Handley CE, Lichtenberger SM, Myck DM. Refusals and delay of immunization within southwest Alberta. Canadian Journal of Public Health 2002: 93(2), 109-112.

(9) Muscat, M. Who gets measles in Europe? J Infect Dis 2011: 204 (Suppl 1), S353-S365.

(10) Knol MJ, Urbanus AT, Swart EM, Mollema L, Ruijs WL, van Binnendijk RS, te Wierik MJ, de Melker HE, Timen A, Hahné SJ. Large ongoing measles outbreak in a religious community in the Netherlands since May 2013. Euro Surveill. 2013; 18(36):pii=20580.

(11) Alberta Health Services. Routine Immunization Schedule. http://www.health.alberta.ca/healthinfo/imm-routine-schedule.html Offit PA, Moser CA. The problem with Dr. Bob's Alternative Vaccine Schedule. Pediatrics 2009; 123:e164-e169.

(12) Lee BR, Feaver SL, Miller CA, Hedberg CW, Ehresmann KR. An elementary school outbreak of varicella attributed to vaccine failure: policy implications. J Infect Dis 2004: 190(3), 477-483.

(13) Parker AA, Staggs W, Dayan GH, et al. Implications of a 2005 measles outbreak in Indiana for sustained elimination of measles in the United States. N Engl J Med 2006; 355:447-455.

\section{Remerciements}

Les auteurs souhaitent remercier Brittany Tyssen, Michelle Davidson et Sjaane Heikoop pour leur aide dans l'analyse des données des écoles, ainsi que l'ensemble du personnel de l'Alberta Health Services et du ministère de la Santé de l'Alberta, qui conserve les dossiers de vaccination.

\section{Conflit d'intérêts}

II n'y a aucun conflit d'intérêts à déclarer.

\section{Financement}

Aucun financement externe n'a été reçu. 